\title{
Autorenverzeichnis 1989
}

Ackermann, R. , 248 Adolph, M.,204, Sl Agternkamp,C.,77,133 Aigner, K. R.,119

Bässler, K. H.,82 Baldermann, H., 233 Balogh,D.,52 Baumert, H., 267 Baumgartl, P.,45

Beger,H. G.,156 Bertholf, M.F., S2 Betzler,H.,125 Bittner, R.,156 Blauhut, B.,167 Bock,W. J.,102 Böck,M.,183 Böhler, S.,82 Böhm, R., S2 Borberg, H., S2 Brenner, U., SI Burchard,W. G.,77,133 Butters, ML, 156

Castor, G., 30 Chapman, R., S2

Daschner, F., 241 Dengler,T.,160 Deurenberg, P., 276 Dietze,G.,233 Dommisch, K., 88

Doran,J.E.,167 Druml,W., Sl Dutcher, J.P., S2

Eckart, J.,204, Sl Eckle, R., S2

Fauth, U.,253, Sl Fellerer,K.,92 Ferdinand, P., 92 Fosburg, M. T., S2 Frank, U., 241 Franke,R. P.,77,133 Freesen, P., 88 Fürst,G.,160 Furtwängler, W. , 52

Gallasch,E.,267,273 Göddecke, W., S2 Gollmer,E.,248 Günther,B.,230

Hach-Wunderle,V.,100 Hackl,J. M.,52 Halmágyi, M., 253, SI Hartig,W.,125 Hautvast, J.G.A.J.,276 Hebenstreit, G. F., 92 Heim, M.U.,183 Heine, W., 264 Heinrichs, W.,253, Sl Herrmann, B., 107 Hester, J., S2 Holbach,T.,30 Holmes, M.,S 2 Hütt,M.,107 Jacobson, M. S., S 2 Jaksche,H.,30 Jarosch, E., 68 Jürgens P., SI Jung,F., 30,148 Kadar,J. G., S2 Kahn,J. M.,52

Kalff, G.,77,133 Keferstein, R., 60 Keller, H. W.,237, SI Kellner, S.,160 Kevy,S. V., S2 Kiesewetter, H.,30,148 Kosswig, W., SI Kretschmer, V., S 2 Krüger,J.,248 Kühnl,P.,100 Kuhrt,E.,88 Kurz,R.,68

Leweling, H., 107 Lundsgaard-Hansen, P., 167 Lynen,R.,267,273

Mahlke,L.,102 Marin,H.,88 Matkowitz, R,, 125

Mehnert, H.,233 Mempel,W.,183 Mertes,N., 60,114,222 Metzger, S., 156 Mintz, P.D., S2

Mittermayer, Ch., 77,133 Moore,R. C., S2 Mory, M.,41 Mrowietz, C, 148 Müller,F. G.,77,133 Müller,H.,119 Müller,J. M.,237, SI Müller,N.,186 Musil,H. E.,68

Nanoff, C.,141 Neeser, G., 204, SI Neumeyer, H., 273 Nolte,G., 60,114,222 Northway, M. M., S2

Pelzer, H., S2

Pfeíffer,P.,92 Pfisterer, M.,60,114 Piek, J.,102 Pinsker, K.,45 Price, T. H., S2 Prinz, H., S2 Puchstein,Ch., 60, 114,222 Puénte-Gonzales, I., 253

Radke,M.,264 Rank, A., S 2 Reinauer, H., SI Reissigl, H.,251 Rett,K.,233 Ritter, R.,125 Ryan, J., S2

Scharrer, I.,IOO Schauder, P.,Sl Schleich,I.,183 Schmidt, R.,248 Schmitz, J.E.,216, SI

Schober,P. H.,68 Schuyler, B., S2 Seidell,J. C.,276 Seufzer, U.,107

Simon, J., 30 Sindu,E.,273 Söhngen, D., S2 Spitzer, S.,148 Staedt, U.,107 Stock, M. J., 282

Stöcker, U.,160 Storm, H., 88

Taborski,U.,186 Teixidor,D.,100 Thul,P.,237 Twerdy, B.,92 Tzanova, I.,253, Sl

Urdahl, S. G., S2

Ventura, G., S 2 
Wagner, B.,S 2 Wagner, K., 82 Wagner, M., 183 Waldhausen, P.,148 Walpot,H.,77,133 Walter, M.,SI , Walther,H.,119 Wehner,W.,41 Weindler, R.,183 Wengert, P.,204 Wenzel, E.,148

Wicklmayr, M.,233 Widhalm,K.,141,199 Willroth,P. 0.,88 Winde,G.,60,114 Wretlind, A.,192 Wutzke,K. D.,264

Zadravec, S.,92 Zander,J., 60,114 Zumpe,P.,100 Zunser, R.,248 Zwiauer,K.,141,176

(Autoren von Abstrakts sind nicht im Jahresgesamtverzeichnis aufgeführt) 